\title{
TEM investigation of MoSeC films
}

\author{
C.Silviu Sandu ${ }^{\mathrm{a}, \mathrm{b}}$, Tomas Polcar ${ }^{\mathrm{a}, \mathrm{c}}$ and Albano Cavaleiro, ${ }^{\mathrm{a},{ }^{*}}$ \\ ${ }^{a}$ ICEMS - Mechanical Engineering Department, University of Coimbra, Polo 2, 3030-788 \\ Coimbra, Portugal \\ ${ }^{\mathrm{b}}$ EPFL - Ecole Polytechnique Federale de Lausanne, CH-1015 Lausanne, Switzerland \\ ${ }^{\mathrm{c}}$ Department of Control Engineering, Faculty of Electrical Engineering, Czech Technical \\ University in Prague, Technická 2, Prague 6, Czech Republic \\ *albano.cavaleiro@dem.uc.pt
}

Transition metal dichalcogenides (TMD) are widely used as self-lubricating material either as oil additive or directly as thin films. Magnetron sputtering is a deposition method allowing depositing such films with high density and adhesion. However, their spread use in practical applications is still hindered since their excellent sliding properties are deteriorated in the presence of humidity and under high contact pressures. $\mathrm{MoSe}_{2}$, one of the members of TMD family recently studied [1], has been co-sputtered with carbon in order to improve the mechanical and tribological properties when compared to pure $\mathrm{MoSe}_{2}$ films.

The films have been deposited by co-sputtering from a $\mathrm{C}$ target embedded with a different number of $\mathrm{MoSe}_{2}$ pellets. Prior to the deposition of Mo-Se-C films a $300 \mathrm{~nm}$ Ti layer was deposited on the steel and Si substrates in order to improve the adhesion. The chemical composition was measured by electron probe microanalysis (EPMA). Cross-sectioned specimens were prepared from samples containing 38, 51 and 68 at.\% of carbon (Se/Mo ratio $1.8,1.8$ and 2.0, respectively) by mechanical polishing and ion milling. The morphology and the chemical composition of the coatings were investigated by HRTEM and EDS in a PhilipsCM300 microscope. To support TEM analysis, the coatings were tested by Raman spectroscopy ( $\mathrm{Ar}^{+}$laser, $514.5 \mathrm{~nm}$ wavelength), X-Ray Photoelectron spectroscopy (XPS $\mathrm{Mg} \mathrm{K} \alpha$ radiation) and X-ray diffraction (XRD - Brag-Brentano configuration in glancing mode, $\mathrm{Co} \mathrm{K} \alpha$ radiation) and their hardness was evaluated by depth sensing indentation (Fisherscope H100, maximum load $50 \mathrm{mN}$ ). The XPS spectra were taken after ion etching with $\mathrm{Ar}+$ ions $\left(3 \mathrm{KeV}\right.$ and $\left.5 \mu \mathrm{A} / \mathrm{cm}^{2}\right)$ during $3 \mathrm{~min}$.

TEM micrographs show that film morphology strongly depends on the $C$ content (Fig. 1). By increasing the $\mathrm{C}$ content of the films, the $\mathrm{MoSe}_{2}$ nanocrystallites became smaller as indicated by the increase of the width of the diffraction rings in SAED patterns. At the same time the film morphology became more homogeneous. The contrast observed in the Mo-Se-C layers, after about $200 \mathrm{~nm}$ thickness, can be attributed to the material porosity, probably related to the typical columnar morphology usually developed in TMD films. The lower part of the films looks more dense and homogeneous (low porosity). The films porosity clearly decreases with increasing $\mathrm{C}$ content (Fig. 1 and 2); simultaneously, the columnar structure almost vanishes. This result complements the information obtained by previous SEM analysis of MoSe-C coatings published in Ref [1], (see the right part of the pictures in figure 1) where the aspect of low density is kept even for high $\mathrm{C}$ content films. Based on TEM analysis, it seems that the columnar growth is typical only for low carbon coatings and the dendritic growth described in Ref [2] and observed in figure 1 is probably only an artifact originated during sample breaking. The increase of the material density could promote the improvement of the hardness from 1.4 to $4.0 \mathrm{GPa}$ with increasing $\mathrm{C}$ content in the range 38-68 at.\%.

The alignment of the basal planes of the $\mathrm{MoSe}_{2}$ phase leads to the formation of platelets which, in contrast images, have the appearance of wires (Figs. 2 and 3). The increase of C 
amount in the layer stops the development of the ordering of the basal $\mathrm{MoSe}_{2}$ planes leading to decreasing platelets dimensions, as can be concluded by the shortening of these apparent wires. Fig. 2a shows long curved chains consisting of tens of parallel $\mathrm{MoSe}_{2}$ basal planes, in contrast to Fig. 2c, where the platelets thickness is shorter (about $5 \mathrm{~nm}$ ) and clearly separated. The most interesting feature in XRD diffractograms of Mo-Se-C coatings is the presence of a highly asymmetric peak, with a long tail towards higher $2 \theta$, positioned at $2 \theta \approx 44.5^{\circ}$ (Fig. $2 \mathrm{~d}$ ). It has been shown [1] that the extended shoulder of the sputtered $\mathrm{MoS}_{2}$ peak positioned close to $2 \theta \approx 40^{\circ}$ corresponds to a turbostrating stacking of $(10 \mathrm{~L})$ planes $(\mathrm{L}=0,1,2,3)$. XRD patterns then could be explained by a $2 \mathrm{D}$ organization of the basal plans which could have several tenths of unit cells dimension. The broadening or drop in the intensity of the (10L) plan is observed with the progressive decrease of the lateral dimensions of the basal plans and the platelets. This situation would arise when the lateral order of the basal plans did not exceed a couple of lattice parameters [3]. It should be pointed out that similar results have been obtained in case of W-S-C films, where the existence of $\mathrm{WS}_{2}$ nanograins in a carbon matrix was confirmed by HRTEM observation [4].

TEM results are confirmed as well by Raman analysis, as documented in Ref [2]. The peak representing $\mathrm{MoSe}_{2}$ phase, positioned at $\sim 240 \mathrm{~cm}^{-1}$, is very broad compared to the one of $\mathrm{MoSe}_{2}$ bulk material indicating a strong degradation of its structural quality. At the same time, almost no change of the carbon peaks (i.e. intensity ratio between $G$ and $D$ peaks and $G$ peak position) is observed, see inset of Fig. 4. It should be pointed out that EDS investigation reveals a lateral and transverse chemical homogeneity of all the analyzed Mo-Se-C films at a $10 \mathrm{~nm}$ scale.

The $\mathrm{MoSe}_{2}$ platelets with diameters of approximately $0.4 \mathrm{~nm}$ appear to be embedded in an amorphous matrix. In spite of the difficulty in proving the assumed nanocomposite structure and to model the chemical bonding of the Mo-Se-C films by TEM analysis, Raman results proved the existence of independent $\mathrm{MoSe}_{2}$ and C-based mixed phases. Furthermore, with high resolution TEM, no traces of molybdenum carbides nanograins could be detected confirming the results of XPS analysis [2]. XPS C1s peak was almost identical, whatever the $\mathrm{C}$ content was, with only a symmetrical peak positioned at $\mathrm{C}-\mathrm{C}$ binding energy and not requiring deconvolution considering any other peak. Also, the presence of molybdenum carbide phases could not be detected by XRD spectra.

In this work, Mo-Se-C coatings deposited by magnetron sputtering from carbon target with different number of $\mathrm{MoSe}_{2}$ pellets were analyzed by TEM. The addition of the $\mathrm{C}$ played a decisive role in the modification of the chemical bonding, morphology and structure of the coating. The formation of a nanocomposite material (nanocrystals of $\mathrm{MoSe}_{2}$ embedded in an amorphous matrix) was possible over a wide range of deposition conditions. The dimension of the crystalline zones seemed to decrease with increasing $\mathrm{C}$ content as could be concluded by the progressive broadening of XRD peaks. The appearance of features like wires in the TEM observations suggested that $\mathrm{MoSe}_{2}$ platelets were embedded in an amorphous carbon matrix.

\section{Acknowledgements}

This work was supported by the Czech Science Foundation through the project 106/07/P014, by the Ministry of Education of the Czech Republic (project MSM 6840770038) and by the Fundação para a Ciência e a Tecnologia through the project SFRH/BPD/34515/2006. CIMEEPFL team is acknowledged for the technical support. 


\section{References}

[1] T. Kubart, T. Polcar, L. Kopecký, R. Novák and D. Nováková, Surf. Coat. Technol. 193 (2005) 230.

[2] T. Polcar et al., Surf. Coat. Technol. (2007), doi:10.1016/j.surfcoat.2007.08.019.

[3] G. Weisse, N. Mattern, H. Hermann, A. Teresiak, I. Bacher, W. Bruckner, H.-D. Bauer, H. Vinzelberg, G. Reiss, U. Kreissig, M. Mader, P. Markschlager, Thin Solid Films 298 (1997) 98.

[4] A. Nossa, A. Cavaleiro, J. Mater. Res. 19 (2004) 2356.
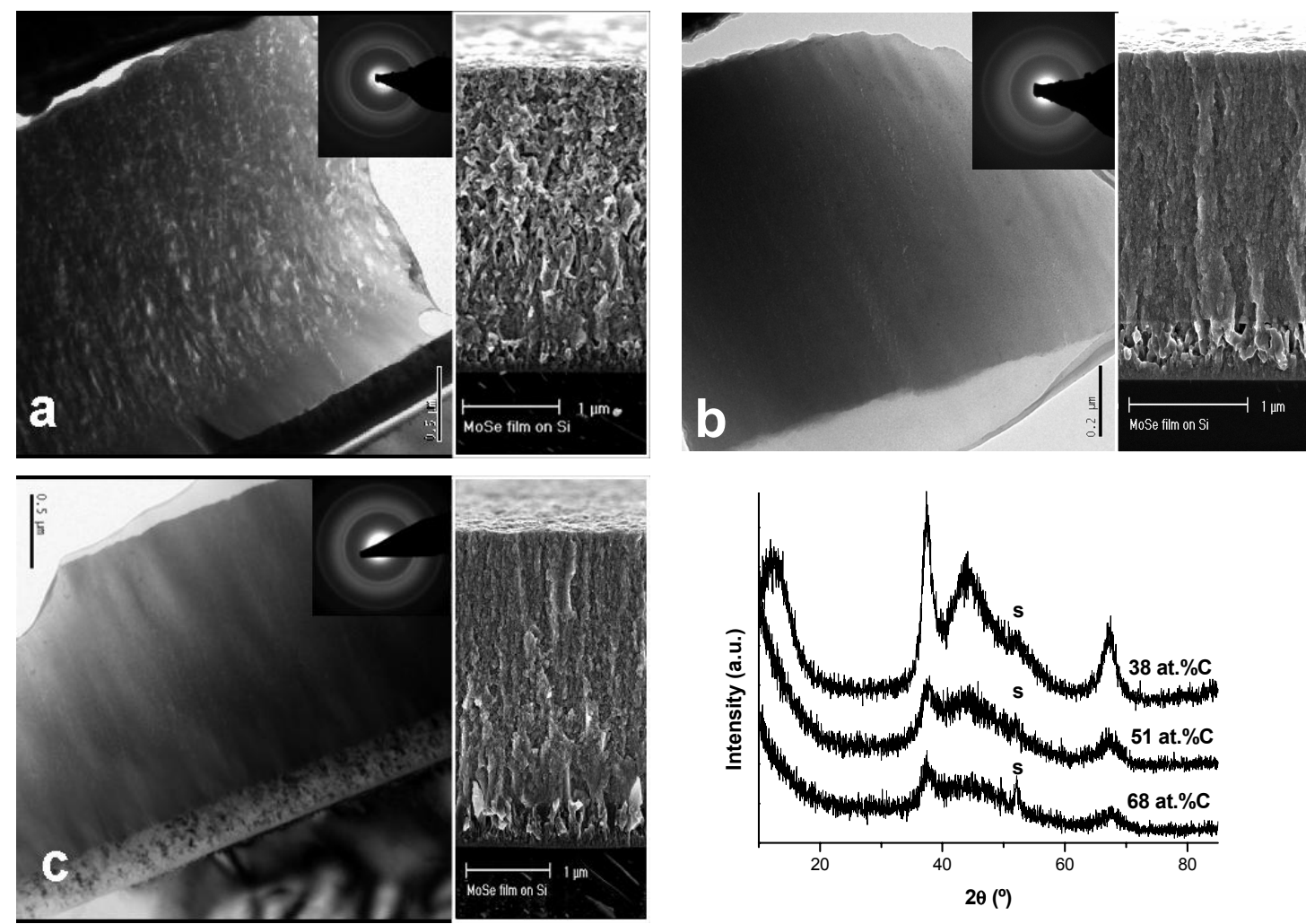

Fig.1 TEM images of MoSeC films cross-sections with corresponding SEM micrographs: a $38 \% \mathrm{C}, \mathrm{b}-51 \% \mathrm{C}, \mathrm{c}-68 \% \mathrm{C}$, and d) XRD diffraction patterns. 

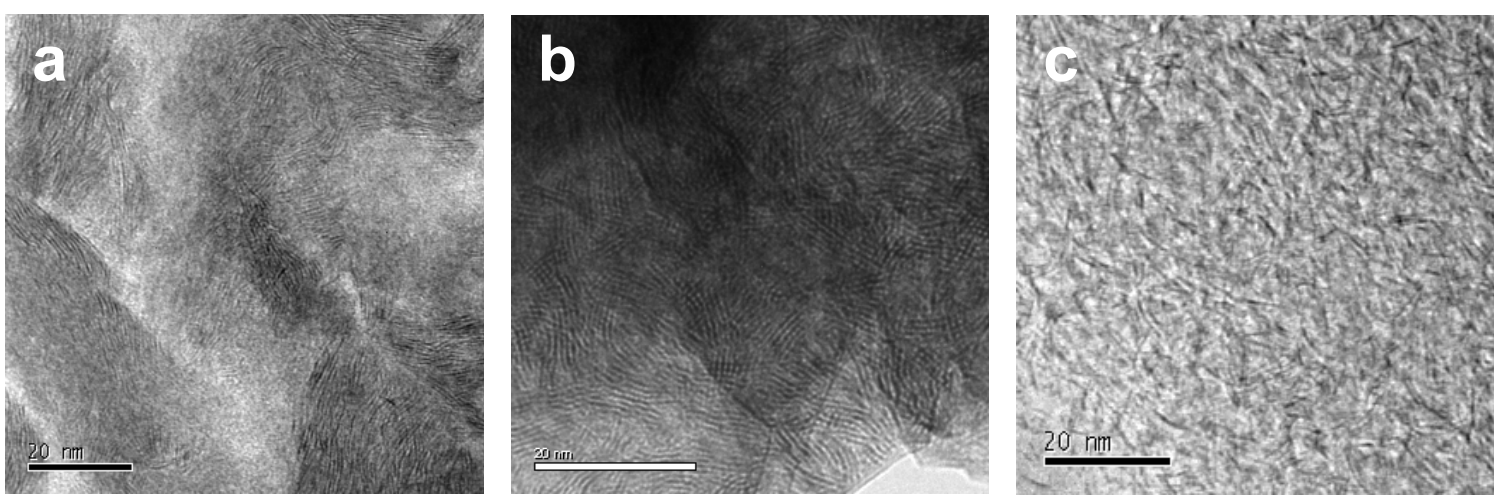

Fig.2 TEM images of Mo-Se-C films: a - 38\% C, b- 51\% C, c - 68\% C.
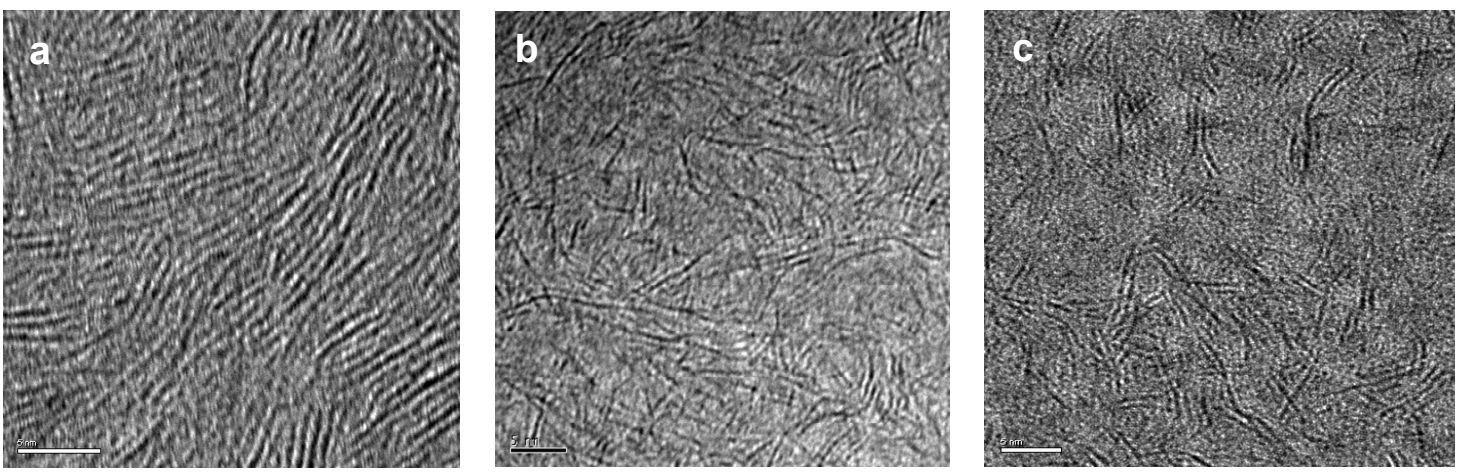

Fig. 3 HRTEM images of Mo-Se-C films: a - 38\% C, b-51\% C, c - 68\% C. 